having witnessed it, I can say nothing, except that it appears to me that it does not replace the various parts in their original position, or give a sufficient support to the perinæum.

\section{THE TREATMENT OF CHRONIC ULCERS.}

By WILLIAM STUART LOW, L.R.C.P.(Edin.), etc., Yealand Conyers.

So much doubt has been expressed, diffidence entertained, and practitioners so frequently stigmatised as rash or timorous, according as they attempt or not to heal chronic ulcers, that a clearer understanding of their treatment and its effects is much to be desired. That there is no danger in the perfect cure of long standing and much discharging ulcerated surfaces, I maintain ; and I cite the following case in evidence.

R. S., male, aged 48, had suffered for twelve years from a very extensive ulceration of the right leg. During this time, a great variety of treatment had been tried-rest, bandaging, baths, and numerous internal medicines. I found the case one of extensive serpiginous ulcer, almost one mass of broken surface on each side of the knee, back of the calf, and popliteal surface, and even extending to the posterior surface of the thigh, at some points granulating, and at others sloughing, bleeding on the slightest injury. The surfaces were deep, and the edges raised and hard, with much surrounding induration. The discharge was very offensive, and this especially so from the grumous pus expressed from numerous sinuous channels ramifying about the limb.

My patient being in easy circumstances, I had full liberty as regards any expense I might incur, and determined to make one last endeavour. I attacked the whole surface by means of a thorough ablution with a lotion of the solution of bichloride of mercury, of the strength of one drachm to eight ounces of water; this was practised daily, syringing most carefully every part of the wound, and with speedy benefit. The discharge rapidly lessened, the foetor diminished, and the sloughing ceased. New granulations sprang up all over, although not without considerable loss of blood from recurrent attacks of hæmorrhage as the dead tissues separated and the feeble granulations gave way. The dressing consisted simply of lint wrung out in the bichloride lotion of similar strength, and applied directly to the wounds.

The second feature in the treatment that I am anxious to emphasise, is the utility of iodoform as a local anæsthetic. This was very marked, relieving the pain sorely complained of in many irritable spots, contributing greatly to the comfort of the patient, and facilitating exceedingly the daily dressing. The iodoform was used freely in the form of a fine powder, dusted upon the painful spots. It proved valuable also in quickly inducing healthy action in the indolent portions of the ulcer, and, as an antiseptic and deodorant, maintained a perfect sweetness of the discharges.

I observed, in the local employment of powdered iodoform, however, that its use must not be persisted in too long, or the healthy action obtained is soon undone, the granulations breaking down under its continued action, and much bloody discharge staining the dressings : indeed, a blood-stained dressing, where iodoform had been applied, became the indication to cease its employment there. While preparing the wound for cicatrisation, by stimulating the surface, it rather retarded the growth of the cuticle, and simple dressing with bichloride lotion proved more efficacious. Lastly, its application to an inflamed surface should be studiously avoided, as it tends greatly to aggravate it.

The beneficial effect of these remedies is more remarkable, when I state that this patient rested his leg but little. Rest was a part of my prescription that he stoutly resisted, daily walking short distances, driving out, and going up-stairs. It was here that the third important point in the treatment proved invaluable; the use of resilient pressure, in the form of a Martin's elastic bandage; this was daily applied over the other dressings, from toes to thigh.

During the interval of six weeks since commencing these measures, the progress has been uninterrupted, and the cure is now complete and I trust such encouraging results will animate many to essay the successful therapeutics of chronic ulceration.

Charbon in French Pork. - Charbon has never been experiment ally communicated to pigs. M. Villain, principal inspector of the Paris butcher-shops, seized some doubtful looking pork offered for sale at the Halles (Central Market). M. Nocard examined it, and detected bacteria of charbon in the blood. A rabbit and guinea-pig were injected with the blood, and both died.

\section{PATHOLOGICAL MEMORANDA.}

\section{CAUSATION OF CARDIAC POLYPI OR THROMBI.}

Is the epitomised version of my remarks upon a case of thrombus in the left ventricle, at the January meeting of the Leeds and West Riding Medico-Chirurgical Society, there are two inaccuracies for which I hope I may disclaim responsibility. The chief mass of the thrombus was not "laid flat on the surface" of the apex of the ventricle, but was interlaced, as well as adherent, in the usual way. Certain detached masses were, however, adherent to even surfaces, and were clearly not at all dependent for their position upon any interlacing. "The interior of the chief (?) cardiac cavities" did not "contain puriform fluid," but only the interior of the cyst-like softening thrombus in the left ventricle contained it.

I ventured to doubt the sufficiency of the ordinary theory of the formation of cardiac polypi, inasmuch as while they are very rare, their supposed cause (feeble or dilated ventricle) is very common. In four cases examined by myself, obvious endo-myocarditis existed in three, with formation of cardiac aneurysm in two of them. In another case (fifth), a mass nearly an inch in length, three-fourths of an inch in breadth, and a quarter of an inch thick, had grown from the auricular surface of the mitral valve, and projected into the auricular cavity. This was of course merely an overgrown "vegetation." Apex-polypi or thrombi appear to me to originate in a similar manner; and their rarity seems due to the rarity of endocarditis in this position.

T. Chunton, M.D. Physician to the Leeds Infirmary.

\section{CLINICAL MEMORANDA.}

\section{MORBID SOMNOLENCE.}

ON January 6th, 1886, about 1 P.M., a gentleman called upon me, asking me to go and see his "domestic," who, he said, had failed to waken that morning, and was now fast asleep. All ordinary means had failed to rouse her. On going to the house I found a stout, florid, healthy girl, apparently about 18 years of age, sleeping, or apparently sleeping, quite calmly. Her mistress told me that the evening before she had suffered from toothache, and that she might have taken an overdose of laudanum. She presented none of the symptoms of narcotic poisoning. I examined the pupils, and found them both markedly dilated. I then shook her, but with no effect. Upon my slapping her cheeks, she put up her hands to cover her face, and began to weep, but other than that she showed no signs of becoming awake. I, with some assistance, got her out of bed, and walked her up and down the floor, when, after a few turns backwards and forwards, she gradually opened her eyes, looked dazed, butshortly came to herself. I ordered her a strong cup of coffee, and after that a cold bath. I asked her to come to me in the evening, when she told me the following story. She is aged 15 , a strong full-blooded country girl. Her family history is good. She herself had gone through the usual infantile ailments, but never had any illness of long duration. She had not yet menstruated, and had never felt any indication of it. She had always lived in the country, and had only come to town about a couple of weeks ago. She complained of nothing; indeed, at the time she was speaking to me, she was not conscious of any ailment, and was quite at a loss to account for her excessive somnolence. About a month ago she felt this extreme drowsiness coming over her, but then thought nothing of it. I found that she had distinct anæsthesia of the right side, and pain under the right breast, and headache; she never had "fits" of any kind. She had never felt this pain so severe as to complain of it, but she had noticed that she was not quite so handy with the right hand as with the left. There was no ovarian pain.

On the morning of January 13th, I was again called to see her, on account of sound sleep. Her mistress had tried the somewhat rough and ready remedies of my former visit, but with no effect. The most remarkable feature of this second attack was that, though seemingly sound asleep, as soon as I spoke to her she cowered; and, on being ordered, in a peremptory tone, to walk about the room, she got out of bed, walked across the floor backwards and forwards, avoiding chairs and tables, turning when I told her, and all the while in an apparent sleep. I washed her face well with cold water, which gradually wakened her. She told me afterwards that she ineard my voice quite distinctly, but could not speak or open her eyes, and, when ordered to walk about, she felt compelled to obey me.

J. Hutchison, M.D., Shawlands, Glasgow. 